\title{
New class of solutions to Laplace equation: Regularized multipoles of negative orders
}

\author{
Matt Majic $\odot$ and Eric C. Le Ru $\odot^{*}$ \\ The MacDiarmid Institute for Advanced Materials and Nanotechnology, School of Chemical and Physical Sciences, \\ Victoria University of Wellington, PO Box 600, Wellington 6140, New Zealand
}

(Received 26 July 2019; published 31 December 2019)

\begin{abstract}
We introduce a new class of solutions to the Laplace equation, dubbed logopoles, and use them to derive a new relation between solutions in prolate spheroidal and spherical coordinates. The main novelty is that it involves spherical harmonics of the second kind, which have rarely been considered in physical problems because they are singular on the entire $z$ axis. Logopoles, in contrast, have a finite line singularity like solid spheroidal harmonics, but are also closely related to solid spherical harmonics and can be viewed as an extension of the standard multipole ladder toward the negative multipolar orders. As part of our derivations, we also found a new integral representation for the spherical harmonics of the second kind in terms of their source distributions. As an example application, we use logopoles to construct a fast converging series solution for the problem of a point charge interaction with a dielectric sphere, which extends the basic image approximations introduced by Kelvin, Kirkwood, and Friedman. We believe these new solutions will prove a fruitful alternative to either spherical or spheroidal harmonics in a wide range of other physical problems.
\end{abstract}

DOI: 10.1103/PhysRevResearch.1.033213

\section{INTRODUCTION}

The Laplace equation is the fundamental equation in a wide range of physical problems including astronomy, geophysics, electrostatics, and fluid mechanics [1-4]. It is also strongly related to the even more pervasive Helmholtz equation, which governs wave phenomena in, for example, acoustic and electromagnetic scattering. The latter link is both mathematical, since the solutions have similarities, and physical, since the Laplace equation is the long-wavelength limit of the Helmholtz equation. The solution of the Laplace equation using the separation of variable method is a standard textbook problem $[1,5]$. In spherical coordinates $(r, \theta, \phi)$, the angular part of the solution consists of spherical harmonics, proportional to $P_{n}^{m}(\cos \theta) e^{i m \phi}$, where $n$ is a positive integer called the multipole order, $m$ is an integer satisfying $|m| \leqslant n$, and $P_{n}^{m}$ is the associated Legendre function of the first kind. The radial part of the solution is of the form $r^{n}$ (finite at the origin) or $r^{-n-1}$ (singular at the origin, but regular at infinity). This results in two types of solutions called solid spherical harmonics (SSHs): the internal SSHs, $r^{n} P_{n}^{m}(\cos \theta) e^{i m \phi}$, and the external SSHs, $r^{-n-1} P_{n}^{m}(\cos \theta) e^{i m \phi}$. A similar approach exists in other coordinate systems, and we will focus here on prolate spheroidal coordinates $(\xi, \eta, \phi)$ defined by two focal points. The angular dependence is similar, of the form $P_{n}^{m}(\eta)$, while the radial solution involves Legendre functions of the first kind $P_{n}^{m}(\xi)$ (finite at the origin), or second kind $Q_{n}^{m}(\xi)$

\footnotetext{
*eric.leru@vuw.ac.nz

Published by the American Physical Society under the terms of the Creative Commons Attribution 4.0 International license. Further distribution of this work must maintain attribution to the author(s) and the published article's title, journal citation, and DOI.
}

(singular at $\xi=1$, segment between the two foci, but regular at infinity). The internal and external prolate spheroidal solid harmonics (PSSHs) $P_{n}^{m}(\xi) P_{n}^{m}(\eta) e^{i m \phi}$ and $Q_{n}^{m}(\xi) P_{n}^{m}(\eta) e^{i m \phi}$ are commonly used in problems with spheroids or elongated objects. Other types of solutions have more recently been studied [6-10], but none as fundamental and generally applicable as SSHs and PSSHs. Interestingly, it was shown recently that PSSHs also provide an advantage in problems with spherical geometry [11], where the SSHs would have a priori been better suited. Links between SSHs and PSSHs via series expansions were derived more than a century ago [12].

Both external SSHs and PSSHs have bounded singularities and go to zero at infinity, making them useful for solving problems outside bounded domains. Although rarely mentioned or used, equivalent solutions exist where the angular part takes the form of the associated Legendre functions of the second kind, $Q_{n}^{m}(\cos \theta)$. These are normally discarded due to their singularities on the entire $z$ axis, which precludes their application to bounded physical systems. We here present a way around this problem by combining SSHs of the second kind centered at two different origins to remove the singularities at infinity. This approach can be understood simply by considering the lowest order solution $Q_{0}(\cos \theta)$. Close to the $z$ axis, it behaves as $Q_{0}(\cos \theta) \sim \ln (z / \rho)$, where $\rho=\sqrt{x^{2}+y^{2}}$, hence the singularity for $\rho=0$. Now let us define two offset coordinate frames with origins $\mathrm{O}^{\prime}$ and $\mathrm{O}^{\prime \prime}$ at $z=R$ and $z=-R$ on the $z$ axis and their associated spherical coordinates $\left(r^{\prime}, \theta^{\prime}, \phi\right)$ and $\left(r^{\prime \prime}, \theta^{\prime \prime}, \phi\right)$, see Fig. 1 . We have for small $\rho$

$$
\begin{aligned}
Q_{0}\left(\cos \theta^{\prime}\right)-Q_{0}\left(\cos \theta^{\prime \prime}\right) & \underset{\rho \rightarrow 0}{\sim} \ln \frac{z-R}{\rho}-\ln \frac{z+R}{\rho}+O\left(\rho^{2}\right) \\
& \sim \underset{\rho \rightarrow 0}{\sim} \ln \frac{z-R}{z+R}+O\left(\rho^{2}\right)
\end{aligned}
$$




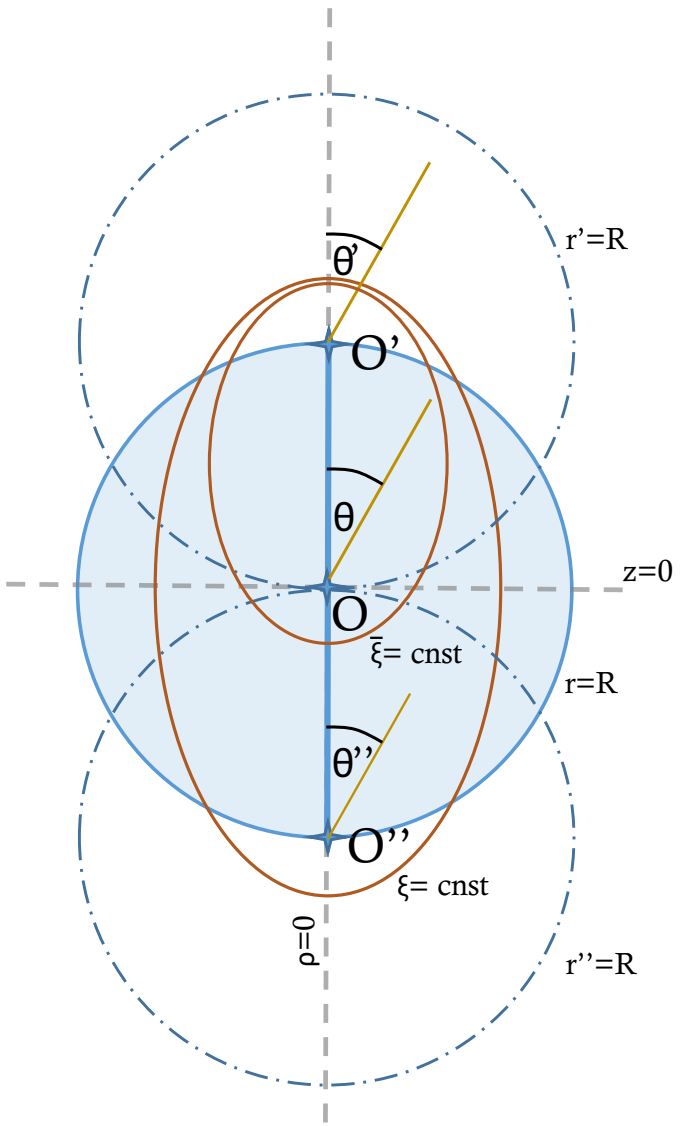

FIG. 1. Schematic of the centered and offset spherical and prolate spheroidal coordinate systems considered in this work.

which is finite except on the segment between $\mathrm{O}^{\prime}$ and $\mathrm{O}^{\prime \prime}$. By combining two offset SSHs of the second kind, we have therefore obtained a solution whose singularity is bounded, which makes such combinations suitable for solving practical problems. Interestingly, the resulting segment singularity is the same as that of the PSSH solutions when $\mathrm{O}^{\prime}$ and $\mathrm{O}^{\prime \prime}$ are chosen as the foci defining the spheroidal coordinates, in fact the solution in Eq. (1) is the PSSH of order $0, Q_{0}(\xi)$ $[11,13]$.

In this work, we explore further this idea. We generalize this simple example to derive a new relationship between the uncommon SSHs of the second kind and the more common PSSHs. To prove this relation, we introduce a new class of solutions, dubbed logopoles, that are closely related to both spherical and spheroidal harmonics. Like the spheroidal harmonics, they are singular on a bounded line segment and are therefore suitable for modeling similar physical problems. We discuss logopole properties, their relationships to spherical and spheroidal harmonics, and possible applications. As an example, we present one practical application of logopoles in constructing a fast converging image series for the problem of a point charge interaction with a dielectric sphere. Our discussion of logopoles will be here restricted to harmonics with $m=0$, but they can be generalized to arbitrary $m$. This generalization, although conceptually similar, is not straightforward and brings added technicalities, so will be presented elsewhere to allow us to focus here on the concepts rather than the mathematics. For the same reason, many secondary proofs are given in the Appendixes.

This work suggests that the SSHs of the second kind and the related logopoles may provide a fruitful alternative to the common SSHs and PSSHs in some problems involving the Laplace equation. The greatest benefits of these concepts could be realized when extending them to other related equations, notably the Helmholtz equation.

\section{NEW RELATIONSHIP INVOLVING SOLID SPHERICAL HARMONICS OF THE SECOND KIND}

We first present a new formula expressing PSSHs as a finite sum of offset SSHs of the second kind at origins $\mathrm{O}^{\prime}$ and $\mathrm{O}^{\prime \prime}$ :

$$
\begin{aligned}
Q_{n}(\xi) P_{n}(\eta)= & \sum_{k=0}^{n} \frac{(n+k) !}{2 k !^{2}(n-k) !(2 R)^{k}} \\
& \times\left[(-1)^{n+k} r^{\prime \prime k} Q_{k}\left(\cos \theta^{\prime \prime}\right)-r^{\prime k} Q_{k}\left(\cos \theta^{\prime}\right)\right] .
\end{aligned}
$$

$P_{n}$ are the Legendre polynomials and $Q_{n}$ the Legendre functions of the second kind. Spherical coordinates $(r, \theta, \phi)$ (with $u \equiv \cos \theta)$ are centered at the origin $\mathrm{O}$, cylindrical coordinates are denoted $(z, \rho, \phi)$, and the offset coordinates (see Fig. 1) can be expressed as

$$
\begin{array}{ll}
\rho^{\prime}=\rho=\sqrt{x^{2}+y^{2}}, & \rho^{\prime \prime}=\rho, \\
z^{\prime}=z-R, & z^{\prime \prime}=z+R, \\
r^{\prime}=\sqrt{\rho^{2}+(z-R)^{2}}, & r^{\prime \prime}=\sqrt{\rho^{2}+(z+R)^{2},} \\
u^{\prime}=\cos \theta^{\prime}=z^{\prime} / r^{\prime}, & u^{\prime \prime}=\cos \theta^{\prime \prime}=z^{\prime \prime} / r^{\prime \prime} .
\end{array}
$$

The prolate spheroidal coordinates $(\xi, \eta, \phi)$ are taken with foci at $\mathrm{O}^{\prime}$ and $\mathrm{O}^{\prime \prime}$ and defined as in Ref. [1]:

$$
\xi=\frac{r^{\prime \prime}+r^{\prime}}{2 R}, \quad \eta=\frac{r^{\prime \prime}-r^{\prime}}{2 R} .
$$

The right-hand side of Eq. (2) is a generalization of the simple example in Eq. (1). It is a carefully chosen combination of offset SSHs of the second kind that ensures their singularities at infinity cancel out. The resulting sum is only singular on the segment from $\mathrm{O}^{\prime}$ to $\mathrm{O}^{\prime \prime}$ and happens to correspond to the PSSHs. It is known that $Q_{n}(\xi) P_{n}(\eta)$ can also be expanded as a series of spherical harmonics of the first kind [12], but the expansion is infinite and only converges outside the sphere of radius $R$ centered at the origin. In contrast, the sum in Eq. (2) is finite and is valid everywhere except on the singularity line from $\mathrm{O}^{\prime}$ to $\mathrm{O}^{\prime \prime}$. Equation (2) raises the prospect of the more general applicability of SSHs of the second kind. Despite the relative simplicity of this expression, we could not find a simple proof, but propose a more indirect proof, which is interesting in its own right as it leads us to introduce new functions: logopoles.

\section{LOGOPOLES}

We define logopoles first through their integral representation, with a source distribution consisting of a finite line of charge extending from $\mathrm{O}$ to $\mathrm{O}^{\prime}$ with charge density $(z / R)^{n}$ :

$$
L_{n}=R \int_{0}^{1} \frac{v^{n} d v}{\sqrt{\rho^{2}+(z-R v)^{2}}} .
$$


As we shall see later, this is similar to the spheroidal harmonics integral representation. But we first manipulate this definition to find closed form expressions and recurrence relations, and in doing so we will see that logopoles are also closely related to spherical harmonics of the second kind. First, it is straightforward to expand logopoles as a series of multipoles, by expanding the denominator in Eq. (3) as a Taylor series in $v$ and integrating each term, giving

$$
L_{n}(\hat{r}, \theta, \phi)=\sum_{k=0}^{\infty} \frac{S_{k}}{n+k+1},
$$

where $S_{n}(\hat{r}, \theta, \phi)=\hat{r}^{-(n+1)} P_{n}(\cos \theta)$ are the external SSHs of the first kind. For convenience, we have defined adimensional "hat" coordinates that are scaled by $R$, for example, $\hat{r}=r / R$. This series diverges for $r<R$ but has the following analytic continuation to all space except the line segment $0 \leqslant z \leqslant R$ on the $z$ axis:

$$
L_{n}=\tilde{S}_{n}-\sum_{k=0}^{n}\left(\begin{array}{l}
n \\
k
\end{array}\right) \tilde{S}_{k}^{\prime},
$$

where $\tilde{S}_{n}(\hat{r}, \theta, \phi)=\hat{r}^{n} Q_{n}(\cos \theta)$ are the "internal" SSHs of the second kind and the prime means that the function is of primed coordinates: $\tilde{S}_{n}^{\prime}=\tilde{S}_{n}\left(\hat{r}^{\prime}, \theta^{\prime}, \phi\right)$. This provides the link with Eq. (2) and suggests that logopoles can be viewed as a regularization of the internal SSHs of the second kind $\left(\tilde{S}_{n}\right)$. The latter is singular on the $z$ axis and diverges at $\infty$, but the finite sum of offset SSHs in Eq. (5) removes this singularity and divergence such that the logopoles are singular only on the $z$ axis between $0 \leqslant z \leqslant R$. Equations (4) and (5) could be used as alternative definitions for the logopoles. We note that logopoles can also be expressed in terms of offset multipoles $S_{n}^{\prime}$ (see Appendix A), or in terms of $L_{0}$ to isolate the logarithmic singularity (see Appendix B). The lowest-order logopoles can be written as

$$
\begin{gathered}
L_{0}=\ln \frac{r^{\prime}-z^{\prime}}{r-z}=\ln \frac{r+z}{r^{\prime}+z^{\prime}}=Q_{0}(\xi), \\
L_{1}=\left[u L_{0}-1\right] \hat{r}+\hat{r}^{\prime}, \\
L_{2}=\frac{1}{2}\left[\left(3 u^{2}-1\right) L_{0}-3 u\right] \hat{r}^{2}+\frac{1}{2}[3 u \hat{r}+1] \hat{r}^{\prime} .
\end{gathered}
$$

Figure 2 presents plots of representative logopoles and PSSHs.

We now sketch a derivation of Eq. (5), which gives further insight into the link between logopoles and multipoles. We start from the well-known expansion of an offset point charge in terms of centered multipoles:

$$
S_{0}^{\prime}=\frac{R}{r^{\prime}}=\sum_{k=0}^{\infty} S_{k},
$$

and note that differentiation along $z$ is a ladder operator for SSHs [14], explicitly

$$
R \partial_{z} S_{n}=-(n+1) S_{n+1} .
$$

For this proof we will use integration instead of differentiation to move down the other way on the multipole
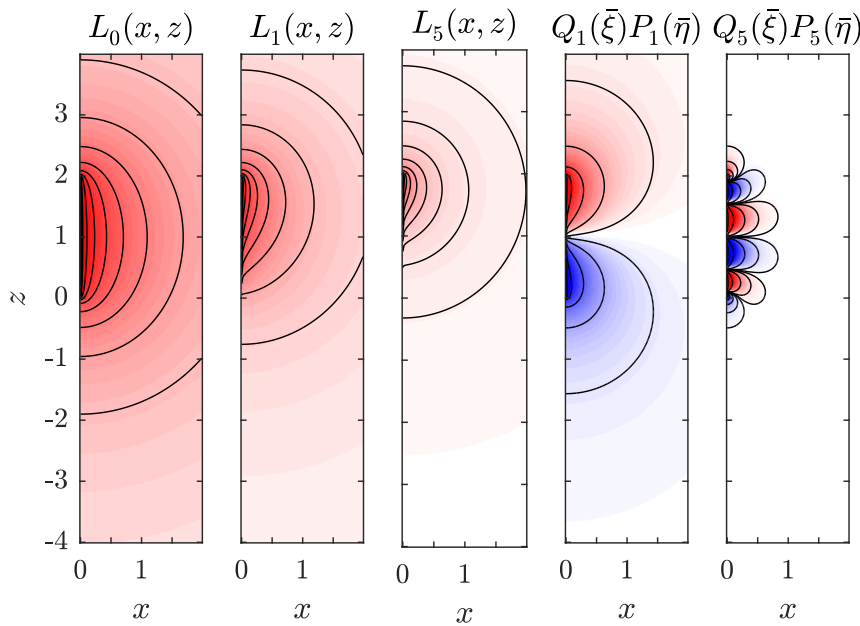

FIG. 2. Intensity plots of a selection of low-order logopoles and offset PSSHs with $R=2$. For better visualization, the functions have been rescaled and transformed by taking the arcsinh, which is similar to plotting on a log scale but allows negative values. Red is positive, blue negative. The solid lines represent equipotentials.

ladder:

$$
\begin{gathered}
\int d \hat{z} S_{n}=-\frac{S_{n-1}}{n}+f_{1}(\rho), \quad n \geqslant 1, \\
\int d \hat{z} S_{0}=\tilde{S}_{0}+f_{2}(\rho),
\end{gathered}
$$

with $f_{i}(\rho)$ arbitrary functions. Equation (11) derives from the ladder operator expression and Eq. (12) is easily checked by integrating explicitly. By integrating Eq. (9), we then obtain

$$
\tilde{S}_{0}^{\prime}=\tilde{S}_{0}-\sum_{k=0}^{\infty} \frac{S_{k}}{k+1}+f(\rho) .
$$

We prove in Appendix $\mathrm{C}$ that $f(\rho)=0$ and recognize the infinite series definition of $L_{0}$, which therefore satisfies $L_{0}=$ $\tilde{S}_{0}-\tilde{S}_{0}^{\prime}$. Since the series in Eq. (13) converges for $z>R$, we must have that $L_{0}$ is also finite there, even though $\tilde{S}_{0}=$ $Q_{0}(\cos \theta)$ and $\tilde{S}_{0}^{\prime}$ are singular on the $z$ axis. This proves Eq. (5) for $n=0$. Logopoles of higher-order $n$ can be obtained through repeated integration with respect to $z$, as shown in Appendix C.

The ladder operator applies to logopoles in a similar way as to SSHs of the second kind:

$$
\begin{gathered}
R \partial_{z} \tilde{S}_{n}=n \tilde{S}_{n-1}, \\
R \partial_{z} L_{n}=n L_{n-1}-S_{0}^{\prime} .
\end{gathered}
$$

Equation (15) may be derived by differentiating the source integral (3); see Appendix D. This simple relation is in stark contrast with that for the spheroidal harmonics, where the operator results in an infinite series [15]:

$$
R \partial_{z} Q_{n}(\xi) P_{n}(\eta)=-2 \sum_{\substack{k=n+1 \\ k+n \text { odd }}}^{\infty}(2 k+1) Q_{k}(\xi) P_{k}(\eta) .
$$


We can also show (see Appendix E) that the logopoles obey the following recurrence relation (for $n \geqslant 1$ ):

$$
(n+1) L_{n+1}-\hat{z}(2 n+1) L_{n}+\hat{r}^{2} n L_{n-1}=\hat{r}^{\prime},
$$

which, up to the inhomogeneous term $\hat{r}^{\prime}$, is identical to the recurrence for $\tilde{S}_{n}$. Again, the PSSHs do not obey a similarly simple recurrence. The recurrences (15) and (17) show that logopoles are in some respect much closer to SSHs than to PSSHs despite having a line singularity like the latter. In addition, the proof by integration along with the radial dependences $\left(r^{n}\right.$ vs $\left.r^{-n-1}\right)$ and Eq. (14) all suggest that the internal SSHs of the second kind can be viewed as the extension of external SSHs of the first kind to $n<0$. Although the link is not rigorous, we can write informally that $\tilde{S}_{n} \equiv S_{-n-1} / 0$ where the division by zero represents what would result if we naively extrapolated Eq. (11) through $n=0$ [one could argue $r^{n} P_{n}(\cos \theta)$ are the SSHs for $n<0$, since $P_{-n-1}=P_{n}$, but these do not fit on the same ladder described by the operator $\partial_{z}$ ]. As $L_{n}$ provide a regularization of $\tilde{S}_{n}$ to ensure the singularity remains bounded, the logopoles can therefore be viewed as the most physical definition for multipoles of negative orders.

\section{LINK BETWEEN LOGOPOLES AND SPHEROIDAL HARMONICS}

We now derive the relations linking logopoles to spheroidal harmonics, from which a proof of Eq. (2) will result. Spheroidal harmonics are known to be proportional to the potential of a charge distribution on a finite line segment, given by the Havelock formula [16,17]:

$$
Q_{n}(\xi) P_{n}(\eta)=R \int_{-1}^{1} \frac{P_{n}(v) d v}{\sqrt{\rho^{2}+(z-R v)^{2}}} .
$$

Since the singularity of the logopoles lies on the segment $\mathrm{OO}^{\prime}$ and that of PSSHs on $\mathrm{O}^{\prime} \mathrm{O}^{\prime \prime}$, we define a translated spheroidal coordinate system with foci at $\mathrm{O}$ and $\mathrm{O}^{\prime}$ denoted $\bar{\xi}, \bar{\eta}$ :

$$
\bar{\xi}=\frac{r+r^{\prime}}{R}, \quad \bar{\eta}=\frac{r-r^{\prime}}{R} .
$$

The integral representation for offset PSSHs is easily derived from Eq. (18):

$$
Q_{n}(\bar{\xi}) P_{n}(\bar{\eta})=\frac{R}{2} \int_{0}^{1} \frac{P_{n}(2 v-1) d v}{\sqrt{\rho^{2}+(z-R v)^{2}}} .
$$

The similarities with the integral representation of logopoles is clear: The singularity is the same and while spheroidal harmonics are produced by Legendre polynomial charge distributions, logopoles are produced by monomial distributions. This link can be made explicit by expanding the Legendre polynomials $P_{n}(x)$ as a series of powers of $(x+1)$ [see Eq. (F5)] and setting $x \equiv 2 v-1$ :

$$
P_{n}(2 v-1)=\sum_{p=0}^{n} \frac{(-)^{n+p}(n+p) !}{p !^{2}(n-p) !} v^{p} .
$$

Substituting in Eq. (20) and recognizing the integral representation of logopoles, we deduce

$$
Q_{n}(\bar{\xi}) P_{n}(\bar{\eta})=\sum_{p=0}^{n} \frac{(-1)^{p+n}(n+p) !}{2 p !^{2}(n-p) !} L_{p} .
$$

Using the inverse relationship of Eq. (21), we also obtain

$$
L_{n}=\sum_{k=0}^{n} \frac{2 n !^{2}(2 k+1)}{(n-k) !(n+k+1) !} Q_{k}(\bar{\xi}) P_{k}(\bar{\eta}) .
$$

We note that these expansions have the same coefficients (up to a factor of 2) as those that relate internal PSSHs $P_{n}(\bar{\xi}) P_{n}(\bar{\eta})$ to internal SSHs $r^{n} P_{n}(\cos \theta)$ [Eq. (A.2) in Ref. [18] for $m=$ $0]$, in which $r^{n} P_{n}(\cos \theta)$ would take the place of $L_{n}$, and $P_{n}(\bar{\xi})$ the place of $Q_{n}(\bar{\xi})$. This is yet another similarity between logopoles and multipoles. We also see that logopoles for $n \leqslant N$ span the same space as offset PSSHs with $n \leqslant N$, providing an alternative basis to that space. We have not been able at this stage to prove or disprove the completeness of the infinite set of $L_{n}$. It is also worth pointing out that since logopoles are positive functions [from Eq. (3)], they cannot satisfy the same orthogonality relations as spheroidal or spherical harmonics.

We can now return to the proof of Eq. (2). Substituting Eq. (5) into Eq. (22) and simplifying using a binomial identity (see Appendix F), we obtain the offset PSSHs as a finite sum of SSHs of the second kind:

$$
Q_{n}(\bar{\xi}) P_{n}(\bar{\eta})=\sum_{k=0}^{n} \frac{(n+k) !}{2 k ! 2(n-k) !}\left[(-1)^{n+k} \tilde{S}_{k}-\tilde{S}_{k}^{\prime}\right] .
$$

This relation is similar to Eq. (2) except that the spheroidal coordinates are offset (singular on $\mathrm{OO}^{\prime}$ ). To find the equivalent relation for normal spheroidal coordinates (singular on $\mathrm{O}^{\prime \prime} \mathrm{O}^{\prime}$ ), we apply the successive transformations $R \rightarrow 2 R$ and $z \rightarrow$ $z+R$, which results in Eq. (2), as required.

\section{INTEGRAL REPRESENTATION FOR SPHERICAL HARMONICS OF THE SECOND KIND}

Finally, given the interpretation of logopoles as regularized SSHs of the second kind, it would be instructive to compare Eq. (3) to the source integral for $\tilde{S}_{n}$. We show in Appendix G that $\tilde{S}_{n}$ may indeed be expressed as a line source distribution on the entire $z$ axis:

$$
\begin{aligned}
\tilde{S}_{n}= & \lim _{a \rightarrow \infty}\left\{\frac{R}{2} \int_{-a}^{a} \frac{\operatorname{sign}(v) v^{n} d v}{\sqrt{\rho^{2}+(z-R v)^{2}}}\right. \\
& \left.-\sum_{\substack{k=0 \\
n-k \text { odd }}}^{n} \frac{a^{n-k}}{n-k} \hat{r}^{k} P_{k}(\cos \theta)\right\} .
\end{aligned}
$$

This new relation explains why these functions are normally neglected from physical analysis: the source for $\tilde{S}_{n}$ is the difference between a divergent line source on the entire $z$ axis and a sum of divergent multipoles from sources at $r=\infty$, both containing infinite charge. Logopoles, in contrast, can be seen as a regularization of the SSHs of the second kind, with an identical charge distribution on the $z$ axis but truncated to 


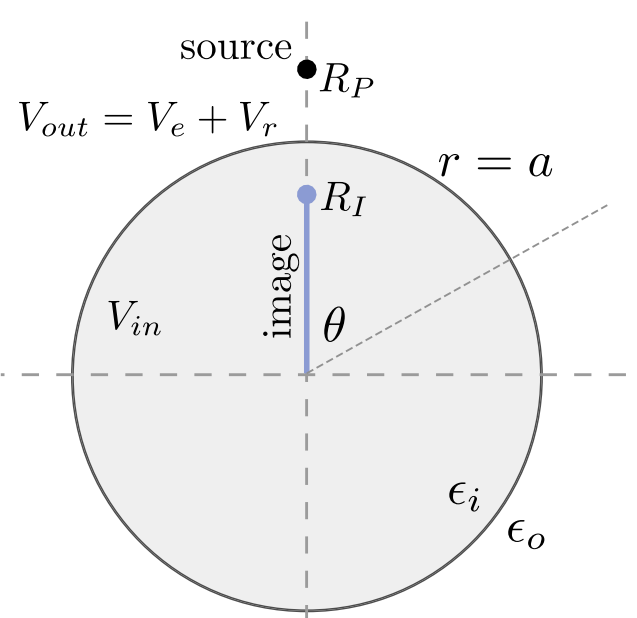

FIG. 3. Schematic of the problem of a point charge interacting with a dielectric sphere.

a finite length. Comparing the integral forms also provides an intuitive explanation for the binomial coefficients occurring in the expansions of logopoles and spheroidal harmonics as finite sums of SSHs of the second kind [Eqs. (5) and (2)]. The coefficients $\left(\begin{array}{l}n \\ k\end{array}\right)$ and $\left(\begin{array}{c}n \\ k\end{array}\right)\left(\begin{array}{c}n+k \\ k\end{array}\right)$ are the same as those arising in the expansion of $v^{n}$ as a binomial expansion in $(v-1)^{k}$, and of $P_{n}(v)$ in powers of $(v+1)^{k}$ and $(v-1)^{k}$ [although this does not show that the diverging finite sums in Eq. (25) cancel].

\section{EXAMPLE APPLICATION: POINT CHARGE INTERACTION WITH DIELECTRIC SPHERE}

We now discuss one possible application of logopoles: to calculate the potential of a point charge near a dielectric sphere. This classic problem can be solved using spherical harmonics [5], but the series converges very slowly when the point charge is close to the sphere [19]. It was recently shown that using PSSHs instead of SSHs could dramatically improve the convergence of the solution [11]. We will show that logopoles provide an even faster converging solution.

Consider a point charge located at $R_{P}$ on the $z$ axis outside a sphere centered at the origin of radius $a$ and permittivity $\epsilon_{i}$ in a medium of permittivity $\epsilon_{o}$, as shown in Fig. 3. The relative permittivity is denoted $\epsilon=\epsilon_{i} / \epsilon_{o}$. Outside the sphere, we write the electric potential as $V_{\text {out }}=V_{e}+V_{r}$ where $V_{e}$ is the exciting potential due to the source charge and $V_{r}$ is the reflected potential due to the sphere. The boundary conditions are continuity of the potential and discontinuity of its derivative:

$$
V_{\text {in }}=\left.V_{\text {out }}\right|_{r=a}, \quad \epsilon \partial_{r} V_{\text {in }}=\left.\partial_{r} V_{\text {out }}\right|_{r=a} .
$$

The problem has a straightforward solution as a series of spherical harmonics [5]:

$$
\begin{gathered}
V_{e}=V_{0} \frac{a}{\sqrt{\rho^{2}+\left(z-R_{P}\right)^{2}}}, \\
V_{r}=-V_{0} \frac{\epsilon-1}{\epsilon+1} \sum_{n=0}^{\infty} \frac{n}{n+\alpha}\left(\frac{R_{I}}{r}\right)^{n+1} P_{n}(\cos \theta),
\end{gathered}
$$

where

$$
\alpha=\frac{1}{\epsilon+1}, \quad R_{I}=\frac{a^{2}}{R_{P}}, \quad V_{0}=\frac{q}{4 \pi \epsilon_{o} a} .
$$

The point $\left(\rho=0, z=R_{I}\right)$ is the inversion point of the source, and is the location of the Kelvin image charge for perfectly conducting sphere. The series (28) converges extremely slowly when the point source is near the surface, hence much work has been done on arranging this solution into more computable forms, where the series is split into similar series with analytic expressions plus a faster converging remainder [20-25].

We propose a similar approach using logopoles. We will choose point $\mathrm{O}^{\prime}$ at the image position to make the link with logopoles and within our previous notations, we therefore have $R \equiv R_{I}$ and $S_{n} \equiv\left(R_{I} / r\right)^{n+1} P_{n}(\cos \theta)$. We then split the series for $V_{r}$ as follows:

$$
\sum_{n=0}^{\infty} \frac{n}{n+\alpha} S_{n}=\sum_{n=0}^{\infty}\left[1-\frac{\alpha}{n+\alpha}\right] S_{n} .
$$

The first term can be identified as the series for an offset point charge at the Kelvin image location:

$$
\sum_{n=0}^{\infty} S_{n}=\frac{R}{r^{\prime}}=\frac{R}{\sqrt{\rho^{2}+(z-R)^{2}}},
$$

and the second term containing $1 /(n+\alpha)$ is actually a logopole of order $\alpha-1$; its line integral expression is given for example in Refs. [26,27]:

$$
L_{\alpha-1}=\int_{0}^{1} \frac{v^{\alpha-1} d v}{\sqrt{\rho^{2}+(z-R v)^{2}}} .
$$

While the various logopole properties are not much help for noninteger orders, we note that the remaining series converges faster than the original series in Eq. (28), and our approach from here will be to continue subtracting terms from the series so that the remainder converges faster. This is similar to the Friedman image approximations [20] which are obtained by expanding $1 /(n+\alpha)$ in powers of $\alpha /(n+1)$; the key is that each successive subtraction leaves the remainder with an extra power of $1 /(n+1)$, increasing the rate of convergence. However, only an analytic expression has been found for the first term only (which in fact is $L_{0}$ ). Instead we propose a more complicated decomposition:

$$
\begin{aligned}
\frac{1}{n+\alpha}= & \frac{1}{n+1}+\frac{1-\alpha}{(n+1)(n+\alpha)} \\
= & \frac{1}{n+1}+\frac{1-\alpha}{(n+1)(n+2)}+\frac{(1-\alpha)(2-\alpha)}{(n+1)(n+2)(n+\alpha)} \\
= & \frac{1}{n+1}+\frac{1-\alpha}{(n+1)(n+2)}+\frac{(1-\alpha)(2-\alpha)}{(n+1)(n+2)(n+3)} \\
& +\frac{(1-\alpha)(2-\alpha)(3-\alpha)}{(n+1)(n+2)(n+3)(n+\alpha)} \\
= & \cdots .
\end{aligned}
$$

Decomposing the series in this more complex way allows us to find closed forms for the subtracted series at every step, not just the first and second. These successive subtractions can 
be viewed as image approximations, and will be expressible in terms of logopoles. The last term with the factor $1 /(n+\alpha)$ converges more quickly as more terms are subtracted. This type of extraction of analytic terms to leave a fast converging remainder is known as a Kummer acceleration or transformation [28]. If this process is continued $K$ times, we have

$$
\frac{n}{n+\alpha}=1-\alpha \sum_{k=0}^{K} \frac{n !(1-\alpha)_{k}}{(n+k+1) !}-\frac{n !(1-\alpha)_{K+1}}{(n+K+1) !} \frac{\alpha}{n+\alpha},
$$

where $(1-\alpha)_{k}=\prod_{p=1}^{k}(p-\alpha)$ is the rising factorial. Putting Eq. (35) back into the the reflected potential [Eq. (28)] and swapping the order of the summation of $n$ and $k$,

$$
\begin{aligned}
V_{r}= & -V_{0} \frac{\epsilon-1}{\epsilon+1}\left[\frac{R_{I}}{r_{I}}-\alpha \sum_{k=0}^{K}(1-\alpha)_{k} \sum_{n=0}^{\infty} \frac{n ! S_{n}}{(n+k+1) !}\right. \\
& \left.-\sum_{n=0}^{\infty} \frac{n !(1-\alpha)_{K+1}}{(n+K+1) !} \frac{\alpha}{n+\alpha} S_{n}\right] .
\end{aligned}
$$

The first series over $n$ in Eq. (36) resembles the series definition for logopoles of order $k$ in terms of offset SSHs [Eq. (A1)]. To make the link, we consider logopoles under the transformation $z \rightarrow R-z$, for which $S_{n}^{\prime}(z \rightarrow R-z)=$ $(-1)^{n} S_{n}$. We then deduce from Eq. (A1) that

$$
\bar{L}_{k} \equiv L_{k}(z \rightarrow R-z)=\sum_{n=0}^{\infty} \frac{n ! k !}{(n+k+1) !} S_{n} .
$$

In effect we have started with the logopole of order $\alpha-1$ in Eq. (30) and expanded it in terms of flipped logopoles up to order $K$, leaving the remainder as a fast converging multipole series. Of course $\bar{L}_{k}$ share analogous properties with $L_{k}$, for example, by transforming Eq. (5), we find closed forms for $\bar{L}_{k}$ :

$$
\bar{L}_{k}=(-1)^{k}\left(\hat{r}^{\prime}\right)^{k} Q_{k}\left(\cos \theta^{\prime}\right)-\sum_{p=0}^{k}(-1)^{p}\left(\begin{array}{l}
k \\
p
\end{array}\right) \hat{r}^{p} Q_{p}(\cos \theta) .
$$

or $\bar{L}_{k}$ can be computed via a recurrence similar to Eq. (17) (although both Eqs. (38) and (17) become significantly numerically unstable for $n \gtrsim 20$ ).

The reflected potential may finally be expressed as a sum of logopoles and spherical harmonics:

$$
\begin{aligned}
V_{r}= & -V_{0} \frac{\epsilon-1}{\epsilon+1}\left[\frac{R_{I}}{r_{I}}-\alpha \sum_{k=0}^{K} \frac{(1-\alpha)_{k}}{k !} \bar{L}_{k}\right. \\
& \left.-\sum_{n=0}^{\infty} \frac{n !(1-\alpha)_{K+1}}{(n+K+1) !} \frac{\alpha}{n+\alpha} S_{n}\right] .
\end{aligned}
$$

We then have a finite series of $K$ terms including logopoles and an infinite series of spherical harmonics. $K$ can in principle be chosen as desired. As $K$ increases, the remaining infinite series converges faster as its terms decrease as $n^{-K-2}$. However, the series of logopoles alone converges slowly as $K$ increases so choosing $K$ too large can be counterproductive. $K$ should be chosen depending on the required degree of

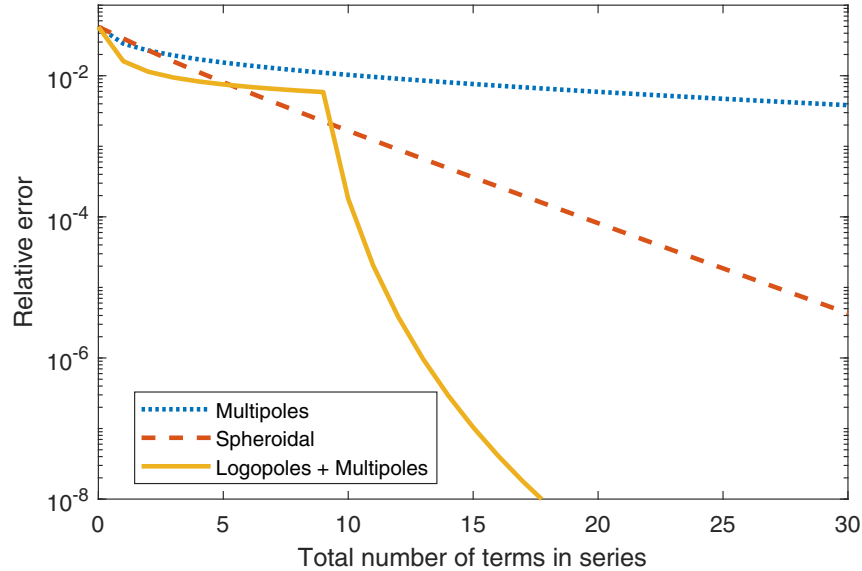

FIG. 4. Comparisons of the relative error in the computed reflected potential of a point charge near a dielectric sphere with dielectric constant 1.5. The charge is located at a distance $0.01 a$ from the sphere, with $a$ its radius, and the reflected potential is calculated at the point charge location using either the spherical harmonic solution (28), the spheroidal harmonic solution from Ref. [11], and the logopole/spherical harmonic solution (39) with $K=10$.

accuracy, which also will depend on the truncation $N$ of the infinite series. We found empirically that to minimize the total number of terms needed to reach a given accuracy, one should use $N \approx K$. This fast convergence is illustrated in Fig. 4 and compared to the series using only spherical or spheroidal harmonics.

There is another way to view this approach. Considering the line charge distribution for $L_{\alpha-1}$ in Eq. (32), we can expand the distribution $v^{\alpha-1}$ in a Taylor series in $(1-v)^{k}$ up to $k=K$. Since the Taylor series is a better approximation near the point $v=1$, the remaining charge distribution becomes more confined to the origin, which intuitively explains the faster convergence of the multipole series.

It is worth noting that the corresponding problem for layered spheres has been recently considered in terms of images [29], and may benefit from a similar approach to that described here.

\section{CONCLUSION AND OUTLOOK}

We conclude by discussing possible implications of this work. Logopoles are potentially interesting in several respects. They are strongly related to spheroidal harmonics through the finite sums in Eqs. (22) and (23) and the common singularity. Given this simple change of basis relating them, one could argue that logopoles may provide no additional benefit over spheroidal harmonics. But we instead believe that both are useful depending on the context, in the same way as one uses Legendre polynomials or monomials for different applications. We have presented one example application, for a point charge near a dielectric sphere, where logopoles are better suited than PSSHs, and we believe more will be found. In addition, in contrast to PSSHs, logopoles have a special link to spherical harmonics and can be viewed as regularized 
multipoles of negative order with many similar properties. We believe this duplicity also makes the logopoles a fruitful concept that deserves further investigation. It moreover highlights the fact that spherical harmonics of the second kind, which are most often neglected from physical analysis, can actually be used to construct localized charge distributions, another concept worth additional investigation. We have generalized this work to the case of a general $m$, and although there are additional mathematical technicalities, the physical interpretation remains similar; this will be presented elsewhere. The application presented here regarding point source interaction with a sphere may also be generalized to dipolar sources using logopoles with $m=1$, and to internal sources and internal fields. Perhaps similar interesting functions can be related to the oblate spheroidal harmonics. More work will also be needed to improve the practical computation of logopoles, since the definitions given here are either numerically unstable in some regions of space for large $n$ or not computationally efficient.

Finally, we speculate that the greatest benefits of these new ideas may come from applying them to the Helmholtz equation $\nabla^{2} V+k^{2} V=0$. The standard solutions in spheroidal coordinates, spheroidal wave functions, are not as user friendly and well behaved as the PSSHs, which render their application much more cumbersome [30]. The ideas developed here could be applied to find better alternatives. For example, our Eqs. (4) and (3) and Eq. A.5 in Ref. [18] can all be generalized to the scalar Helmholtz equation:

$$
\begin{gathered}
H_{n}^{(1)}=\sum_{k=0}^{\infty} \frac{h_{k}(k r) P_{k}(\cos \theta)}{n+k+1}, \\
H_{n}^{(2)}=R \int_{0}^{1} \frac{v^{n} d v e^{i k \sqrt{\rho^{2}+(z-R v)^{2}}}}{\sqrt{\rho^{2}+(z-R v)^{2}}}, \\
H_{n}^{(3)}=\sum_{k=n}^{\infty} \frac{k !^{2} h_{k}(k r) P_{k}(\cos \theta)}{2(k-n) !(k+n+1) !},
\end{gathered}
$$

with $h_{k}$ the spherical Hankel functions. These are just a few possible alternatives to spheroidal wave functions, with identical line singularity as suggested from their long-wavelength limit. These could provide simpler or more efficient alternatives for the solution of wave-scattering problems by spheroidal and elongated objects. For all these reasons, we believe that logopoles and related functions will become a fundamental tool of mathematical physics, alongside multipoles and spheroidal harmonics.

\section{ACKNOWLEDGMENTS}

The authors are grateful to Baptiste Auguié and Dmitri Schebarchov for insightful discussions. We also thank an anonymous reviewer for many constructive comments, including the suggestion to start with Eq. (3) as the definition of logopoles instead of Eq. (4), which simplified several proofs, as well as giving another interpretation of the image extractions in Sec. VI. E.C.L.R. acknowledges the support of the Royal Society Te Apārangi (New Zealand) through a Marsden Grant No. VUW1618.

\section{APPENDIX A: EXPRESSING LOGOPOLES AS A SERIES OF OFFSET MULTIPOLES}

As an alternative series definition to Eq. (4), we can also express $L_{n}$ as a series of SSHs in the offset frame centered on $\mathrm{O}^{\prime}$ :

$$
L_{n}=\sum_{k=0}^{\infty}(-1)^{k} \frac{n ! k !}{(n+k+1) !} S_{k}^{\prime}, \quad r^{\prime}>R,
$$

which is proved below.

The spherical solid harmonics can be expanded on an offset basis at $\mathrm{O}^{\prime}[31]$ :

$$
S_{k}=\sum_{p=k}^{\infty}(-)^{k+p}\left(\begin{array}{l}
p \\
k
\end{array}\right) S_{p}^{\prime}
$$

Inserting this into the original series definition of logopoles [Eq. (4)] and rearranging,

$$
L_{n}=\sum_{p=0}^{\infty} S_{p}^{\prime} \sum_{k=0}^{p} \frac{(-)^{k+p}}{n+k+1}\left(\begin{array}{l}
p \\
k
\end{array}\right)=\sum_{p=0}^{\infty} \frac{(-)^{p} n ! p !}{(n+p+1) !} S_{p}^{\prime}
$$

Here we used a binomial transform identity: Eq. 4.4 in Ref. [32] for $y \equiv n+1$.

\section{APPENDIX B: EXPRESSING LOGOPOLES $L_{n}$ IN TERMS OF $L_{0}$}

While the expression of logopoles in terms of offset SSHs of the second kind, Eq. (5), is the analytic continuation of the logopoles in all space, it is not obvious that the logopoles are finite on the $z$ axis for $z>R$ and $z<0$. To show this, we can express the Legendre functions as $Q_{n}=P_{n} Q_{0}-W_{n-1}$, where

$$
W_{n-1}(x)=\sum_{k=1}^{n} \frac{P_{k-1}(x) P_{n-k}(x)}{k}
$$

is a polynomial of degree $n-1$ [33]. We then use the translation relation for internal spherical harmonics [31]

$$
\sum_{k=0}^{n}\left(\begin{array}{l}
n \\
k
\end{array}\right) \hat{r}^{\prime k} P_{k}\left(u^{\prime}\right)=\hat{r}^{n} P_{n}(u)
$$

to isolate the logarithmic part in Eq. (5):

$$
L_{n}=\hat{r}^{n}\left[P_{n}(u) L_{0}-W_{n-1}(u)\right]+\sum_{k=0}^{n}\left(\begin{array}{l}
n \\
k
\end{array}\right) \hat{r}^{\prime k} W_{k-1}\left(u^{\prime}\right) .
$$

The singularity on $0<z<R$ is then entirely contained within $L_{0}$. This equation also provides a relatively simple analytic expression for the lowest orders of the logopoles.

\section{APPENDIX C: PROOF OF EQ. (5), ANALYTIC CONTINUATION OF LOGOPOLES}

\section{Proof by induction}

Starting from the series definition of logopoles Eq. (4), we aim to prove their analytic continuation as a finite sum of offset spherical harmonics of the second kind Eq. (5), 
explicitly:

$$
\tilde{S}_{n}-\sum_{p=0}^{n} \frac{n ! \tilde{S}_{p}^{\prime}}{p !(n-p) !}=\sum_{k=0}^{\infty} \frac{S_{k}}{k+n+1},
$$

which will be proved by induction on $n$. The base case was covered in the main text. Now assume Eq. (C1) is valid for case $n$, we then integrate with respect to $z$ and re-index the summations to get up to an arbitrary function $f(\rho)$ :

$$
\begin{aligned}
& \frac{\tilde{S}_{n+1}}{n+1}-\sum_{p=0}^{n} \frac{n ! \tilde{S}_{p+1}^{\prime}}{(p+1) !(n-p) !} \\
& \quad=\tilde{S}_{0}-\sum_{k=1}^{\infty} \frac{S_{k-1}}{k(k+n+1)}+f(\rho) \\
& \quad \Rightarrow \tilde{S}_{n+1}-\sum_{p=1}^{n} \frac{(n+1) ! \tilde{S}_{p}^{\prime}}{p !(n+1-p) !} \\
& \quad=\tilde{S}_{0}-\sum_{k=0}^{\infty}\left[\frac{S_{k}}{k+1}-\frac{S_{k}}{k+n+2}\right]+f(\rho) .
\end{aligned}
$$

The sum of $S_{k} /(k+1)$ can be simplified using Eq. (13), and rearranging gives case $n+1$ up to some $f(\rho)$.

\section{Proof of $f(\rho)=0$}

First observe that $f(\rho)$ must be a solution of the Laplace equation because it is a sum of other solutions. The Laplace equation for $f(\rho)$ is simply $\partial_{\rho}\left[\rho \partial_{\rho} f\right]=0$, which has the general solution $f=f_{0} \ln \left(\rho / \rho_{0}\right)$. Then by showing that $f(0)=0$ it follows that $f(\rho)=0$. To show this we will evaluate Eq. (C1) at $\rho=0, z>R$. The right-hand side of Eq. (C1) is

$$
\operatorname{RHS}(\mathrm{C} 1)=\sum_{k=0}^{\infty} \frac{\hat{r}^{-k-1}}{n+k+1} .
$$

For the left-hand side we use the equivalent Eq. (B3), which can be used on the $z$ axis without the problem of $\tilde{S}_{n}$ being singular on the entire axis. For $\rho=0, z>R$, we have $u=$ $u^{\prime}=1, \hat{r}^{\prime}=\hat{r}-1$, and $W_{n-1}(1)=H_{n}=\sum_{k=1}^{n} 1 / k$, the $n$th harmonic number. And $L_{0}$ is expressed using Eq. (4) for $n=0$.

\section{$\operatorname{LHS}(\mathrm{C} 1)$}

$$
\begin{aligned}
& =\hat{r}^{n}\left[L_{0}-H_{n}\right]+\sum_{k=0}^{n}\left(\begin{array}{l}
n \\
k
\end{array}\right)(\hat{r}-1)^{k} H_{k} \\
& =\sum_{k=0}^{\infty} \frac{\hat{r}^{n-k-1}}{k+1}-\hat{r}^{n} H_{n}+\sum_{k=0}^{n} \sum_{p=0}^{k}\left(\begin{array}{l}
k \\
p
\end{array}\right)\left(\begin{array}{l}
n \\
k
\end{array}\right) H_{k}(-)^{k+p} \hat{r}^{p} \\
& =\sum_{q=-n}^{\infty} \frac{\hat{r}^{-q-1}}{n+q+1}+\sum_{p=0}^{n-1} \sum_{k=p}^{n}\left(\begin{array}{l}
n \\
k
\end{array}\right)\left(\begin{array}{l}
k \\
p
\end{array}\right) H_{k}(-)^{k+p} \hat{r}^{p},
\end{aligned}
$$

We then use the identity (proved below)

$$
\sum_{k=p}^{n}(-)^{k+p}\left(\begin{array}{l}
n \\
k
\end{array}\right)\left(\begin{array}{l}
k \\
p
\end{array}\right) H_{k}=\frac{-1}{n-p} \quad(p<n),
$$

to obtain

$$
\begin{aligned}
\operatorname{LHS}(\mathrm{C} 1) & =\sum_{q=-n}^{\infty} \frac{\hat{r}^{-q-1}}{n+q+1}-\sum_{p=0}^{n-1} \frac{\hat{r}^{p}}{n-p} \\
& =\sum_{q=-n}^{\infty} \frac{\hat{r}^{-q-1}}{n+q+1}-\sum_{q=-n}^{-1} \frac{\hat{r}^{-q-1}}{n+q+1}=\operatorname{RHS}(\mathrm{C} 1) .
\end{aligned}
$$

This proves that $f(0)=0$ and therefore $f(\rho)=0$.

\section{Proof of auxiliary identity, Eq. (C3)}

Equation (C3) can be proved by induction on $p$. The base case for $p=0, n>0$ is Eq. 9.3a in Ref. [32] and is also proven in Ref. [34]. Assuming the identity is valid for a given $p$ and all $n>p$, we consider the $n+1, p+1$ cases. We will use the recurrence property of the harmonic numbers: $H_{k+1}=H_{k}+1 /(k+1)$. Then

$$
\begin{aligned}
& \sum_{k=p+1}^{n+1}(-)^{k}\left(\begin{array}{c}
n+1 \\
k
\end{array}\right)\left(\begin{array}{c}
k \\
p+1
\end{array}\right) H_{k} \\
& =-\sum_{k=p}^{n}(-)^{k}\left(\begin{array}{l}
n+1 \\
k+1
\end{array}\right)\left(\begin{array}{l}
k+1 \\
p+1
\end{array}\right)\left[H_{k}+\frac{1}{k+1}\right] \\
& =-\frac{(-)^{p}}{n-p} \frac{n+1}{p+1}-\frac{1}{p+1} \sum_{k=p}^{n}(-)^{k}\left(\begin{array}{l}
n+1 \\
k+1
\end{array}\right)\left(\begin{array}{l}
k \\
p
\end{array}\right),
\end{aligned}
$$

where we used $\left(\begin{array}{c}k+1 \\ p+1\end{array}\right)=\frac{k+1}{p+1}\left(\begin{array}{l}k \\ p\end{array}\right)$ and Eq. (C3). Now we derive another identity to simplify the sum over $k$, by applying the binomial theorem to $x^{n+1}$ twice:

$$
\begin{aligned}
x^{n+1} & =\sum_{k=0}^{n+1}\left(\begin{array}{c}
n+1 \\
k
\end{array}\right)(x-1)^{k} \\
& =1+\sum_{k=0}^{n}\left(\begin{array}{l}
n+1 \\
k+1
\end{array}\right)(x-1)^{k}(x-1) \\
& =1+\sum_{k=0}^{n}\left(\begin{array}{l}
n+1 \\
k+1
\end{array}\right) \sum_{p=0}^{k}\left(\begin{array}{l}
k \\
p
\end{array}\right)(-)^{k+p} x^{p}(x-1) .
\end{aligned}
$$

By rearranging the order of summation and matching the coefficients of each power of $x$, it must be that

$$
\sum_{k=p}^{n}\left(\begin{array}{l}
n+1 \\
k+1
\end{array}\right)\left(\begin{array}{l}
k \\
p
\end{array}\right)(-)^{k+p}=1 .
$$

Insert this into Eq. (C4) to show that the case $n+1, p+1$ holds.

\section{APPENDIX D: PROOF OF EQ. (15), LADDER-TYPE DERIVATIVE OF LOGOPOLES.}

Starting from the source integral (3), we want to show that

$$
R \frac{\partial}{\partial z} \int_{0}^{1} \frac{v^{n} d v}{\sqrt{\rho^{2}+(z-R v)^{2}}}=n \int_{0}^{1} \frac{v^{n-1} d v}{\sqrt{\rho^{2}+(z-R v)^{2}}}-\frac{R}{r^{\prime}} .
$$


First we take the derivative inside the integral and recognize it is equivalent to differentiating with respect to $v$ as follows:

$$
\int_{0}^{1} v^{n} d v R \frac{\partial}{\partial z} \frac{1}{\sqrt{\rho^{2}+(z-R v)^{2}}}=-\int_{0}^{1} v^{n} d v \frac{\partial}{\partial v} \frac{1}{\sqrt{\rho^{2}+(z-R v)^{2}}} .
$$

Then by integration by parts $\int u d w=u w-\int w d u$ with $u=v^{n}, w=\partial_{v}\left(\rho^{2}+(z-R v)^{2}\right)^{-1 / 2} d v$ gives the desired result.

\section{APPENDIX E: PROOF OF EQ. (17), RECURRENCE RELATION FOR LOGOPOLES}

This can be derived from the integral definition, Eq. (3). Consider

$$
\int_{0}^{1} \frac{d}{d v}\left(\frac{v^{n}}{R} \sqrt{\rho^{2}+(z-R v)^{2}}\right) d v
$$

For $n>0$, this integrates to $1^{n} \sqrt{\rho^{2}+(z-R)^{2}} / R=\hat{r}^{\prime}$ by the fundamental theorem of calculus. Alternatively, expanding the derivative in the integrand and rearranging leads to

$$
\int_{0}^{1} \frac{n v^{n-1}\left(\rho^{2}+z^{2}\right)-(2 n+1) z r v^{n}+(n+1) R^{2} v^{n+1}}{R \sqrt{\rho^{2}+(z-v)^{2}}} d v
$$

which, using Eq. (3) for $n-1, n$, and $n+1$, can be recognized as the left-hand side of the recursion relation (17).

\section{APPENDIX F: PROOF OF EQ. (24), EXPANSION OF OFFSET SPHEROIDAL HARMONICS IN TERMS OF SPHERICAL HARMONICS OF SECOND KIND}

We first substitute Eq. (5) into Eq. (22):

$$
Q_{n}(\bar{\xi}) P_{n}(\bar{\eta})=\sum_{k=0}^{n} \frac{(n+k) !}{2 k !^{2}(n-k) !}\left[(-)^{n+k} \tilde{S}_{k}-\sum_{p=0}^{k}(-)^{n+k}\left(\begin{array}{l}
k \\
p
\end{array}\right) \tilde{S}_{p}^{\prime}\right] .
$$

The double sum can be simplified by swapping the summation order to

$$
\Sigma \Sigma=\sum_{p=0}^{n} \tilde{S}_{p}^{\prime} \sum_{k=p}^{n}(-)^{n+k}\left(\begin{array}{l}
k \\
p
\end{array}\right) \frac{(n+k) !}{2 k !^{2}(n-k) !} .
$$

Using the following identity, which we will prove below,

$$
\sum_{k=p}^{n} \frac{(-)^{k}(n+k) !}{k !(k-p) !(n-k) !}=\frac{(-)^{n}(n+p) !}{p !(n-p) !}
$$

we deduce

$$
\Sigma \Sigma=\sum_{p=0}^{n} \frac{(n+p) !}{2 p !^{2}(n-p) !} \tilde{S}_{p}^{\prime}
$$

Substituting back into Eq. (F1), and re-indexing $k \rightarrow p$, we obtain Eq. (24) as required.

To prove the combinatorial identity [Eq. (F3)], we start from the expansions of $P_{n}(x)$ in powers of $(x+1)$ and $(x-1)$ :

$$
\begin{aligned}
P_{n}(x) & =\sum_{p=0}^{n} \frac{(-)^{n+p}(n+p) !}{p !^{2}(n-p) !} \frac{(x+1)^{p}}{2^{p}} \\
& =\sum_{k=0}^{n} \frac{(n+k) !}{k !^{2}(n-k) !} \frac{(x-1)^{k}}{2^{k}} .
\end{aligned}
$$


Then expressing $[(x-1) / 2]^{k}$ as a binomial series of $(x+1) / 2$ :

$$
P_{n}(x)=\sum_{k=0}^{n} \frac{(n+k) !}{k !^{2}(n-k) !} \sum_{p=0}^{k}(-)^{k+p}\left(\begin{array}{l}
k \\
p
\end{array}\right) \frac{(x+1)^{p}}{2^{p}} .
$$

Rearranging the summation order, then equating each coefficient of $(x+1)^{p}$ in Eqs. (F5) and (F7) gives the required identity Eq. (F3).

\section{APPENDIX G: PROOF OF EQ. (25), LINE INTEGRAL FORM FOR SPHERICAL HARMONICS OF THE SECOND KIND}

First of all, informally the charge distribution $\operatorname{sign}(v) v^{n}$ can be obtained from the behavior $r^{n}$ near the $z$ axis and the antisymmetry of $Q_{n}(\cos \theta)$ about $z$. But we will prove Eq. (25) more formally by recurrence using

$$
(n+1) \tilde{S}_{n+1}=(2 n+1) \hat{r} u \tilde{S}_{n}-n \hat{r}^{2} \tilde{S}_{n-1} .
$$

The base cases $n=0,1$ can be obtained from direct evaluation of the integral, by splitting the integration as $\int_{-a}^{a} \operatorname{sign}(v)=$ $\int_{0}^{a}-\int_{-a}^{0}$. Now substituting the assumed integrals for $\tilde{S}_{n}, \tilde{S}_{n-1}$,

$$
\begin{aligned}
(n+1) \tilde{S}_{n+1}= & \lim _{a \rightarrow \infty}\left\{\frac{2 n+1}{2} \hat{r} u \int_{-a}^{a} \frac{\operatorname{sign}(v) v^{n} d v}{\sqrt{\hat{r}^{2}-2 \hat{r} u v+v^{2}}}-\frac{n}{2} \hat{r}^{2} \int_{-a}^{a} \frac{\operatorname{sign}(v) v^{n-1} d v}{\sqrt{\hat{r}^{2}-2 \hat{r} u v+v^{2}}}-\sum_{\substack{k=0 \\
n-k \text { odd }}}^{n-1} \frac{a^{n-k}}{n-k} \hat{r}^{k+1}(2 n+1) u P_{k}\right. \\
& \left.+\sum_{\substack{k=0 \\
n-k \text { even }}}^{n-2} \frac{a^{n-1-k}}{n-1-k} \hat{r}^{k+1} n P_{k}\right\} .
\end{aligned}
$$

We will show that the right-hand side leads to Eq. (25) for $\tilde{S}_{n+1}$. The two integrals can be dealt with identity 2.263.1 of [35] with $n \rightarrow 0, m \rightarrow n$, and re-indexing $k \rightarrow k-1$ in the sum for $\tilde{S}_{n-1}$. This gives

$$
(n+1) \tilde{S}_{n+1}=\lim _{a \rightarrow \infty}\left\{\frac{n+1}{2} \int_{-a}^{a} \frac{\operatorname{sign}(v) v^{n+1} d v}{\sqrt{\hat{r}^{2}-2 \hat{r} u v+v^{2}}}-\frac{1}{2}\left[\sqrt{\hat{r}^{2}-2 \hat{r} u v+v^{2}} v^{n}\right]_{-a}^{a}-\sum_{\substack{k=0 \\ n-k \text { odd }}}^{n-1} \frac{a^{n-k}}{n-k} \hat{r}^{k+1}\left[(2 n+1) u P_{k}-n P_{k-1}\right]\right\} .
$$

In this proof for convenience we use the convention $P_{n}=0$ if $n<0 . \sqrt{\hat{r}^{2}-2 \hat{r} u v+v^{2}}$ can be expanded as a series by integrating the generating function for the Legendre polynomials.

$$
\sqrt{\hat{r}^{2}-2 \hat{r} u v+v^{2}}=\sum_{k=-1}^{\infty} \frac{\hat{r}^{k+1}}{v^{k}} \frac{P_{k-1}-P_{k+1}}{2 k+1} .
$$

Substituting in the bounds and noting that in the limit $a \rightarrow \infty$, we can ignore terms in Eq. (G4) that lead to negative powers of $a$ :

$$
\frac{1}{2}\left[\sqrt{\hat{r}^{2}-2 \hat{r} u v+v^{2}} v^{n}\right]_{-a}^{a}=\sum_{\substack{k=-1 \\ n-k \text { odd }}}^{n-1} a^{n-k} \hat{r}^{k+1} \frac{P_{k-1}-P_{k+1}}{2 k+1} .
$$

Substituting this in Eq. (G3), using the recurrence relation for the Legendre polynomials, and rearranging gives the required expression for $(n+1) \tilde{S}_{n+1}$.

[1] P. M. Morse and H. Feshbach, Methods of Theoretical Physics (McGraw-Hill, New York, 1953).

[2] J. D. Jackson, Classical Electrodynamics, 3rd ed. (Wiley, New York, 1998).

[3] F. D. Stacey and P. M. Davis, Physics of the Earth, 4th ed. (Cambridge University, Cambridge, England, 2008).
[4] N. K. Pavlis, S. A. Holmes, S. C. Kenyon, and J. K. Factor, The development and evaluation of the Earth Gravitational Model 2008 (EGM2008), J. Geophys. Res.: Solid Earth 117, B04406 (2012).

[5] J. A. Stratton, Electromagnetic Theory (McGraw-Hill, New York, 1941). 
[6] P. Garabedian, Orthogonal harmonic polynomials, Pacific J. Math. 3, 585 (1953).

[7] J. Martinek and H. P. Thielman, New solutions of the Laplace equation in spherical coordinates, J. Math. Mech. 116, 1177 (1967).

[8] E. I. Burstein, One of the solutions for the Laplace equation and its physical interpretation, Celestial Mech. 11, 79 (1975).

[9] G. R. Harp and R. S. Sorbello, Unusual solutions of the Laplace equation, Am. J. Phys. 58, 366 (1990).

[10] N. Martinov, D. Ouroushev, and A. Grigorov, New class solutions of the 3-D Laplace equation, J. Math. Phys. 33, 822 (1992).

[11] M. R. A. Majić, B. Auguié, and E. C. Le Ru, Spheroidal harmonic expansions for the solution of Laplace's equation for a point source near a sphere, Phys. Rev. E 95, 033307 (2017).

[12] G. B. Jeffery, The relations between spherical, cylindrical, and spheroidal harmonics, Proc. London Math. Soc. s2-16, 133 (1916).

[13] R. J. Rowley, Finite line of charge, Am. J. Phys. 74, 1120 (2006).

[14] M. Van Gelderen, The shift operators and translations of spherical harmonics, DEOS Progress Letter 98, 57 (1998).

[15] R. L. Matcha, R. H. Pritchard, and C. W. Kern, Prolatespheroidal expansions of the spin-orbit, spin-spin, and orbitorbit operators, J. Math. Phys. 12, 1155 (1971).

[16] T. H. Havelock, The moment on a submerged solid of revolution moving horizontally, Q. J. Mech. Appl. Math. 5, 129 (1952).

[17] T. Miloh, The ultimate image singularities for external ellipsoidal harmonics, SIAM J. Appl. Math. 26, 334 (1974).

[18] M. R. A. Majić, B. Auguié, and E. C. Le Ru, Laplace's equation for a point source near a sphere: Improved internal solution using spheroidal harmonics, IMA J. Appl. Math. 83, 895 (2018).

[19] F. L. B. Da Silva, B. Jönsson, and R. Penfold, A critical investigation of the Tanford-Kirkwood shceme by means of Monte Carlo simulations, Protein Sci. 10, 1415 (2001).

[20] H. L. Friedman, Image approximation to the reaction field, Mol. Phys. 29, 1533 (1975).
[21] R. Abagyan and M. Totrov, Biased probability monte carlo conformational searches and electrostatic calculations for peptides and proteins, J. Mol. Biol. 235, 983 (1994).

[22] S. Deng, W. Cai, and D. Jacobs, A comparable study of image approximations to the reaction field, Comput. Phys. Commun. 177, 689 (2007).

[23] P. Linse and L. Lue, Green's function for a spherical dielectric discontinuity and its application to simulation, J. Chem. Phys. 140, 044903 (2014).

[24] P. D. Rančić, A point ground electrode in the presence of spherical ground inhomogenity: Analysis of two approximate closed form solutions for electrical scalar potential, in Proc. International PhD Seminar Computational Electromagnetics and Technical Applications, Banja Luka, B\&H, 28 August-1 September 2006, pp. 213-223, http://www.phd.etfbl.net/files/ Works_PDF/Rancic\%20Predrag.pdf.

[25] A. Moroz, Superconvergent representation of the GerstenNitzan and Ford-Weber nonradiative rates, J. Phys. Chem. C 115, 19546 (2011).

[26] L. Poladian, General theory of electrical images in sphere pairs, Q. J. Mech. Appl. Math. 41, 395 (1988).

[27] I. V Lindell, Electrostatic image theory for the dielectric sphere, Radio Sci. 27, 1 (1992).

[28] E. E. Kummer, Eine neue methode, die numerischen summen langsam convergirender reihen zu berechnen, J. für die Reine und Angewandte Mathematik 16, 206 (1837).

[29] M. Ma, Z. Gan, and Z. Xu, Ion Structure Near A Core-Shell Dielectric Nanoparticle, Phys. Rev. Lett. 118, 076102 (2017).

[30] N. V. Voshchinnikov and V. G. Farafonov, Optical properties of spheroidal particles, Astrophys. Space Sci. 204, 19 (1993).

[31] E. W. Hobson, The Theory of Spherical and Ellipsoidal Harmonics (Cambridge University, Cambridge, England, 1931).

[32] K. N. Boyadzhiev, Notes on the Binomial Transform (World Scientific, Singapore, 2018).

[33] M. Abramowitz and I. A. Stegun, eds., Handbook of Mathematical Functions (Dover, New York, 1972).

[34] J. Choi, Certain summation formulas involving harmonic numbers and generalized harmonic numbers, Math. Comput. Model. 218, 734 (2011).

[35] I. S. Gradshteyn and I. M. Ryzhik, Table of Integrals, Series, and Products (Academic, New York, 2014). 